\title{
Checklist of the fish fauna of the Araçá Bay, São Sebastião Channel, northern coast of São Paulo, Brazil
}

\author{
Rafael Andrei Lamas, Carmen Lúcia Del Bianco Rossi-Wongtschowski and Riguel Feltrin Contente* \\ Universidade de São Paulo, Instituto Oceanográfico, Departamento de Oceanografia Biológica, Praça do Oceanográfico, 191, CEP05508- \\ 120, São Paulo, SP, Brazil \\ * Corresponding author. E-mail: riguel.contente@gmail.com
}

\begin{abstract}
This paper presents for the first time a checklist of the fish fauna of Araçá Bay, São Sebastião Channel, northern coast of São Paulo state, Brazil. Fishes were sampled in five surveys from October 2012 to February 2014 using nine different types of sampling gear during high tide. Tide pool fishes were also sampled in four surveys from March to October 2014. Geographic distribution and conservation status of each species are reported. A total of 126 species was recorded in Araçá Bay, including two non-native species and new records in the São Sebastião region for 16 species. The fish species richness found in Araçá Bay was greater than the previously recorded for the São Sebastião Channel and other adjacent areas. Most of the Araçá fish species show a wide distribution along the western Atlantic Ocean (32\%). Few species are included in the international (29\%) and Brazilian (3.3\%) official lists of threatened species.
\end{abstract}

Key words: species inventory; species richness; geographic distribution; conservation status; multi-gear approach

\section{INTRODUCTION}

Araçá Bay is a large tidal flat ecosystem located in São Sebastião Channel (SSC) on the northern coast of São Paulo state, adjacent to the São Sebastião Port (Figure 1). It harbors an exceptionally rich macrofauna, including one of the last relicts of mangrove on the São Sebastião coast (Amaral et al. 2010). The port was built in 1936, and since then, its operation, expansion and consequent surrounding urbanization have severely affected the bay's biodiversity (Migotto et al. 1993; Zanardi et al. 1999; Amaral and Nallin 2011). Furthermore, in the 1980s, a submarine sewer was built in the bay, which has caused drastic changes on its morphology and circulation (Teodoro et al. 2011; Amaral et al. 2010; Migotto et al. 1993). Currently, there is a project to expand the São
Sebastião Port toward the bay. A loss of habitat and an increase in water pollution is expected along with the port expansion, and these are expected to affect the bay's ecosystem and consequently its fish community (Consultoria Paulista de Estudos Ambientais 2011).

Several studies of the benthic fauna have been carried out at Araçá Bay (Migotto et al. 1993; Arruda and Amaral 2003; Petracco et al. 2013; Corte et al. 2014; Gorman et al. 2015), but the fish fauna of this bay is still largely unknown, despite its high importance for local fishermen. Available information of the fish fauna is restricted to bottom-trawl surveys (Muto et al. 2000; Rossi-Wongtschowski et al. 2008) and diving surveys (Gibran and Moura 2012) conducted in São Sebastião Channel and on the adjacent continental shelf.

Checklists of species are essential for effective management of ecosystems, as well as for development of conservation plans and environmental impact studies (Silveira 2011; Mace 2004; Hellman and Fowlle 1999). Additionally, comprehensive local inventories may increase our knowledge of the distribution ranges of species and thus provide support to biogeographical and macroecological studies (Silveira et al. 2010). Here, we provide a checklist of fishes from Araçá Bay. We include their geographic distributions and conservation status. The efficiency of the sampling gear used here, and the spatial and temporal variability in the species composition and abundance of the bay will be addressed in future studies.

\section{MATERIALS AND METHODS}

Araçá Bay is composed by four sand beaches, two small islets, three small mangrove patches and a large sand-mud sediment tidal plain. It is limited northward by a rockfill and southward by rocky shores (Figure 1 ). The maximum depth of the bay is $10 \mathrm{~m}$ at its mouth, becoming shallower towards its inner part. At high tide during neap tides, the mean depth from the inner part of the bay to the islets is $0.73 \pm 0.25 \mathrm{~m}$ (SD), and from 

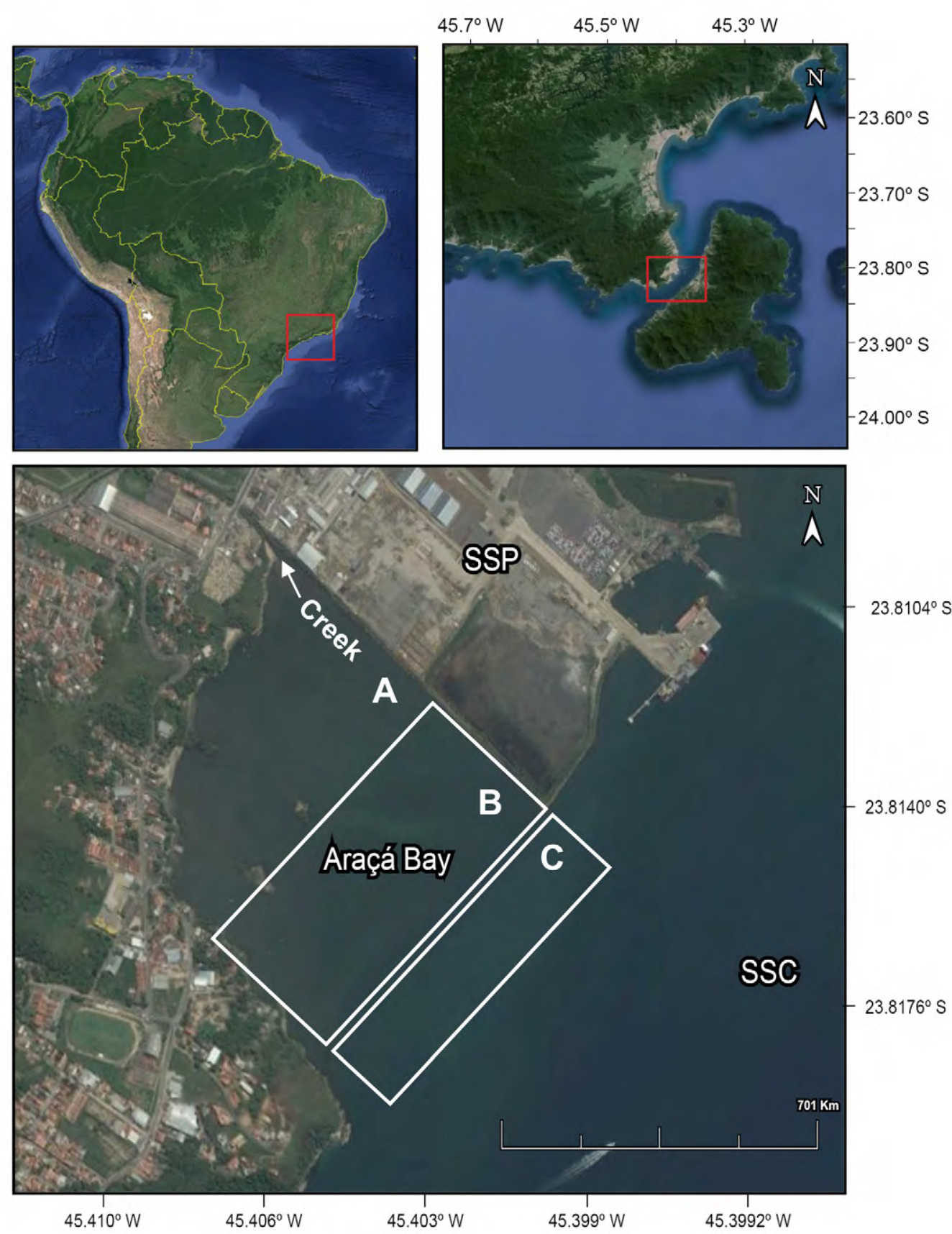

Figure 1. Map of the Araçá Bay at the continental margin of the São Sebastião Channel (SSC), northern coast of São Paulo, Brazil. The arrow indicates Mãe Isabel Creek. São Sebastião Island (SSI); São Sebastião Port (SSP). A: intertidal; B: shallow sublittoral; C: outer sublittoral.

the islets to the bay's mouth is of $1.16 \pm 0.53 \mathrm{~m}$. The bay's configuration prevents it from being directly influenced by the relatively strong SSC hydrodynamics, thus, the main abiotic driver in the bay is the tide (Amaral et al. 2010; Gubitoso et al. 2008).

For our purposes, we divided the bay into three sectors: intertidal, shallow sublittoral and outer sublittoral (Figure 1). Fishes were collected in five surveys: October 2012, March, July, October 2013 and January 2014. The nine types of gear used in each survey and the number and location of deployments are described below:

1. One cast-net $(6.65 \mathrm{~m}$ diameter with a $10 \mathrm{~mm}$ monofilament mesh between adjacent knots) was used in both intertidal and shallow sublittoral sectors, with 18 random and independent deployments in each sector.

2. One otter trawl (two paired nets each with $9.5 \mathrm{~m}$ opening, $6.7 \mathrm{~m}$ cod-end, and $10 \mathrm{~mm}$ between adjacent knots) was operated in the shallow and outer sublittoral, performing four and three random and independent $100 \mathrm{~m}$ tows, respectively.

3. Two encircling gillnets were deployed; one $(590 \mathrm{~m}$ long, $3 \mathrm{~m}$ high, and $30 \mathrm{~mm}$ stretched mesh) was used in the shallow sublittoral with two random and independent deployments; another encircling gillnet (220 $\mathrm{m}$ long, $2.6 \mathrm{~m}$ hight, and $32 \mathrm{~mm}$ stretched mesh) was used in the intertidal, with one deployment at Mãe Isabel creek (Figure 1), and two deployments in the inner sublittoral, one near the rocky shore located south 
of the inner sublittoral and another near the port.

4. Two gillnets, with net $1(154 \mathrm{~m} \times 2.6 \mathrm{~m}$ with $32 \mathrm{~mm}$ stretched mesh) deployed between the two inlets in the intertidal, and net 2 (longer part $=154 \mathrm{~m} \times 3.3 \mathrm{~m}$ and smaller part $=85.8 \mathrm{~m} \times 3.15 \mathrm{~m} ; 50 \mathrm{~mm}$ stretched mesh at both parts) deployed in the southern part of the inner sublittoral from the shore (Figure 1). Each net was operated over 12 hours ( 7 p.m. to $~ 7$ a.m.), being checked at each 2 hours.

5. Six fish traps $(150 \mathrm{~cm}$ wide $\times 53 \mathrm{~cm}$ long $\times 37 \mathrm{~cm}$ high, $15 \mathrm{~mm}$ monofilament line mesh) deployed over the rocky bottom in the southern part of the outer sublittoral.

6. One beach seine ( $20 \mathrm{~m}$ long $\times 3 \mathrm{~m}$ high, with a $15 \mathrm{~m}$ long bag) performing 4 to 5 tows in the margins of the intertidal and 1 to 3 tows in the margins of the inner subtidal.

7. One set of handline ( 15 to 20 size 6 hooks baited with sardines) used over the rocky bottom of the southern part of the outer sublittoral (Figure 1).

One night was required to operate each gear (except for the fish trap, which was continuously operated throughout the day and night over five successive days, and the handline, which was operated in the daytime from approximately 5:00 a.m. to 10:00 a.m.). After the captures, fishes were stored in ice and transported to the laboratory to be identified.

Fishes inhabiting tide pools were sampled during low tide of spring tide in four surveys: March, June, August, and October 2014. The fishes were captured (I) with hand net in the rock and rock-sand pools after dissolving a clove oil solution ( $4 \%$ in alcohol); and (II) with a mosquito-screen-made beach seine $(2.5 \mathrm{~m}$ long, $3.0 \mathrm{~mm}$ mesh) and hand nets in soft-sediment pools (for more details on the sampling procedure, see Brenha-Nunes et al. 2016). All specimens were preserved in $70 \%$ alcohol solution and later identified in laboratory.

The taxonomic identity of the specimens was identified based on Figueiredo (1977), Figueiredo and Menezes (1978, 1980 and 2000), Menezes and Figueiredo (1980 and 1985) and on Carpenter (2002a, 2002b and 2002c). The taxonomic classification follows Eschmeyer (2014). The Chico Mendes Institute for Biodiversity Conservation granted permission for the capture and transportation of the fishes (Permit No. 5574607). Species vouchers are deposited in the Zoological Museum of the University of São Paulo. Voucher numbers are presented in the Appendix, Table A1.

The species were ranked according to the biogeographical categories proposed by Passos et al. (2012), as follow: CT - Circumtropical (throughout the tropics); TA - Trans-Atlantic (western and eastern sides of the subtropical and tropical Atlantic Ocean); WA - Western Atlantic (western side of the subtropical and tropical North and South Atlantic Ocean, with the northern subtropical limit at South Carolina, USA); SWA - Southern West Atlantic (northern Brazil to Argentina); SSWA - Southern South West Atlantic (southeastern Brazil to Argentina); $\mathrm{Ca}$ - Caribbean (Florida, USA, to Venezuela); $\mathrm{Br}$ - Brazilian Province (between the Orinoco delta, Venezuela, and Santa Catarina, Brazil); EA - Eastern Atlantic (eastern side of the subtropical and tropical North and South Atlantic Ocean); and EP - Eastern Pacific (eastern side of the subtropical and tropical Pacific Ocean). Several species have a geographic distribution that does not fit exactly with the biogeographical categories proposed. For such cases, we combined two or three biogeographical categories in order to comply with their distribution.

The conservation status of species was verified in the 'IUCN Red List of Threatened Species' (IUCN 2014), the Brazilian federal list for threatened species, Ordinance of the Ministry of Environment $\mathrm{n}^{\circ} 445$, December 17 of 2014 (Brasil 2014), and the São Paulo state list for threatened species, Decree No 60.133 of February 7 of 2014 (Governo do Estado de São Paulo 2014).

\section{RESULTS}

A total of 126 species were captured, including five elasmobranches (rays) and 121 teleosts belonging to 19 orders and 54 families (Table 1). Photographs of these species were already published by RossiWongtschowski et al. (2015). The richest order was Perciformes (69 spp.), followed by Clupeiformes (9 spp.), and Pleuronectiformes (9 spp.). The richest families were Carangidae (11 spp.), Sciaenidae (9 spp.), Haemulidae, Gobiidae and Paralichthydae (6 spp. each), and Engraulidae and Gerreidae (5 spp. each). Two nonnative, Indo-Pacific species, Omobranchus punctatus (Valenciennes, 1836) (Contente et al. 2015) and Butis koilomatodon (Bleeker, 1849) (Contente et al. 2016), were recorded.

When compared to another comprehensive species checklist for the São Sebastião region (Lamas, 2015), 16 species captured in Araçá Bay were recorded for the first time from the region: Achirus lineatus (Linnaeus, 1758), Astroscopus y-graecum (Cuvier, 1829), Ctenogobius smaragdus (Valenciennes, 1837), Elops saurus Linnaeus, 1766, Gobionellus stomatus Starks, 1913, Hemicaranx amblyrhynchus (Cuvier, 1833), Lutjanus cyanopterus (Cuvier, 1828), Microgobius meeki Evermann \& Marsh, 1899, Mugil hospes Jordan \& Culver, 1895, Myrophis punctatus Lütken, 1852, Omobranchus punctatus, Butis koilomatodon, Sphoeroides testudineus (Linnaeus, 1758), Strongylura marina (Walbaum, 1792), Trachinocephalus myops (Forster, 1801), and Tylosurus acus (Lacepède, 1803).

Most of the species from Araçá Bay (32\%) are largely distributed throughout the subtropical and tropical Western Atlantic Ocean, while 25\% belong to the 


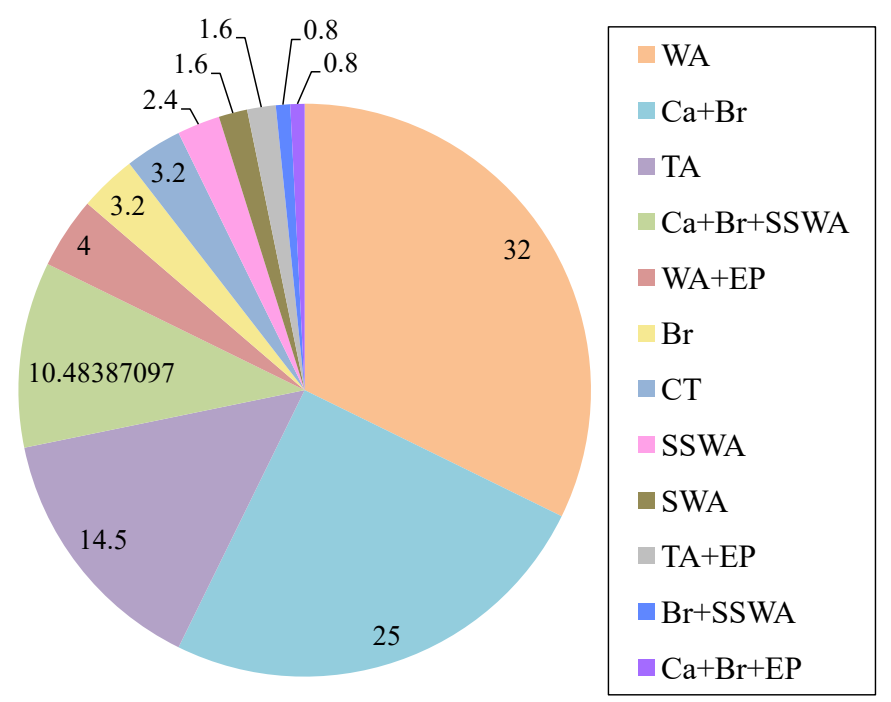

Figure 2. Relative frequency of the geographic distribution categories of species recorded in Araçá Bay, São Sebastião, SP (Total richness = 126). CT: Circumtropical; TA:Trans-Atlantic; WA: Western Atlantic; SWA: Southern West Atlantic; SSWA: Southern South West Atlantic; Ca: Caribbean; Br: Brazilian Province; EA: Eastern Atlantic; EP: Eastern Pacific.

Caribbean and Brazilian Province (a combination of the $\mathrm{Ca}$ and $\mathrm{Br}$ categories). A total of $3.2 \%$ species is restricted to the Brazilian Province, and $2.4 \%$ are distributed along the southern part of the Western Atlantic (Figure 2).

The conservation status of fish species from Araçá Bay is shown in the Table 1 , and the relative frequency of each category is shown in the Figure 3. Only 29\% of the species are ranked within one of the IUCN Red List categories: four species are Data Deficient (DD), 26 are Least Concern (LC), two are Near Threatened (NT; Albula vulpes (Linnaeus, 1758) and Rhinobatos percellens (Walbaum, 1792)), three are Vulnerable (VU; Gymnura altavela (Linnaeus, 1758), Lutjanus analis (Cuvier, 1828) and Lutjanus cyanopterus), and one species is Endangered (EN; Epinephelus marginatus (Lowe, 1834)). According to the Brazilian federal list, only four species are assessed with a Red List category: two species are VU (Hippocampus reidi Ginsburg, 1933 and Lutjanus cyanopterus), and two species are Critically Endangered (CR; Epinephelus marginatus and Gymnura altavela). On the other hand, according to the state list, approximately $50 \%$ of species are included in some category: 38 species are ranked as DD, eight as Fisheries Management Plan Required (FMPR; Epinephelus marginatus, Hippocampus reidi, Lutjanus analis, Lutjanus cyanopterus, Micropogonias furnieri (Desmarest, 1823), Mugil liza Valenciennes, 1836, Rhinobatos percellens and Sardinella brasiliensis (Steindachner, 1879)), and nine as NT (Anchoviella lepidentostole (Fowler, 1911), Centropomus parallelus Poey, 1860, Centropomus undecimalis (Bloch, 1792), Cynoscion jamaicensis (Vaillant \& Bocourt, 1883), Menticirrhus americanus (Linnaeus, 1758), Opisthonema oglinum (Linnaeus, 1758), Orthopristis ruber (Cuvier, 1830), Pomatomus saltatrix (Linnaeus, 1766) and Selene setapinnis (Mitchill, 1815)).

\section{DISCUSSION}

The fish fauna of Araçá Bay is composed of the same pool of species found in other areas along the southeastern Brazilian coast, such as Sepetiba Bay, Rio de

Table 1. Fish species recorded in Araçá Bay, São Sebastião Channel, northern coast of São Paulo, Brazil. Geographic distribution categories (GD); conservation status of the species: the IUCN Red List of Threatened Species (IUCN 2014), the Ordinance of the Ministry of Environment $n^{\circ} 445$, of 17 December 2014 (BR) (Brasil 2014) and the Decree No 60.133 of 7 February 2014 (SP) (Governo do Estado de São Paulo 2014). No geographic distribution category was attributed for Omobranchus punctatus, and Butis koilomatodon since they are originally from the Indo-Pacific. Abbreviations of geographic distribution and conservation status are in figure 2 and 3, respectively. ${ }^{*}$ Species for which vouchers are not available, due to problems in the preservation of the exemplars.

\begin{tabular}{|c|c|c|c|c|c|c|}
\hline Species & Local common names & English common names & Distribution & IUCN & BR & SP \\
\hline \multicolumn{7}{|l|}{ ELASMOBRANCHII } \\
\hline \multicolumn{7}{|l|}{ TORPEDINIFORMES } \\
\hline \multicolumn{7}{|l|}{ Narcinidae } \\
\hline Narcine brasiliensis (Olfers, 1831)* & Raia-elétrica, Treme-treme & Brazilian Electric Ray & WA & DD & $\mathrm{NE}$ & $\mathrm{DD}$ \\
\hline \multicolumn{7}{|l|}{ RAJIFORMES } \\
\hline \multicolumn{7}{|l|}{ Rhinobatidae } \\
\hline Rhinobatos percellens (Walbaum, 1792) & Raia-viola & Chola Guitarfish & TA & NT & $\mathrm{NE}$ & NFMP \\
\hline \multicolumn{7}{|l|}{ MyLIOBATIFORMES } \\
\hline \multicolumn{7}{|l|}{ Dasyatidae } \\
\hline Dasyatis guttata (Bloch \& Schneider, 1801) & Raia-lixa, Raia-branca & Longnose Stingray & $\mathrm{Ca}+\mathrm{Br}$ & DD & $\mathrm{NE}$ & $\mathrm{DD}$ \\
\hline Dasyatis hypostigma Santos \& Carvalho, 2004 & Raia-manteiga, Raia-prego & Bluntnose Stingray & SSWA & DD & $\mathrm{NE}$ & $\mathrm{DD}$ \\
\hline \multicolumn{7}{|l|}{ Gymnuridae } \\
\hline Gymnura altavela (Linnaeus, 1758)* & Raia-amarela, Raia-borboleta & Spiny Butterfly Ray & TA & VU & $\mathrm{CR}$ & $\mathrm{DD}$ \\
\hline \multicolumn{7}{|l|}{ ACTINOPTERYGII } \\
\hline \multicolumn{7}{|l|}{ ELOPIFORMES } \\
\hline \multicolumn{7}{|l|}{ Elopidae } \\
\hline Elops saurus Linnaeus, 1766 & Ubarana & Ladyfish & $\mathrm{Ca}+\mathrm{Br}$ & LC & $\mathrm{NE}$ & NE \\
\hline \multicolumn{7}{|l|}{ Albuliformes } \\
\hline \multicolumn{7}{|l|}{ Albulidae } \\
\hline Albula vulpes (Linnaeus, 1758) & Ubarana-focinho-de-rato & Bonefish & CT & NT & $\mathrm{NE}$ & $\mathrm{NE}$ \\
\hline
\end{tabular}


Table 1. Continued.

\begin{tabular}{|c|c|c|c|c|c|c|}
\hline Species & Local common names & English common names & Distribution & IUCN & BR & SP \\
\hline \multicolumn{7}{|l|}{ ANGULIFORMES } \\
\hline \multicolumn{7}{|l|}{ Muraenidae } \\
\hline Gymnothorax ocellatus Agassiz, 1831 & Moréia, Moréia-pintada & Ocellated Moray & $\mathrm{Ca}+\mathrm{Br}$ & $\mathrm{NE}$ & $\mathrm{NE}$ & NE \\
\hline \multicolumn{7}{|l|}{ Ophichthidae } \\
\hline Myrophis punctatus Lütken, 1852 & Moréia, Enguia & Speckled Worm-Eel & WA & $\mathrm{NE}$ & $\mathrm{NE}$ & $\mathrm{NE}$ \\
\hline Ophichthus gomesii (Castelnau, 1855) & Peixe-cobrado-mar & Shrimp Eel & WA & $\mathrm{NE}$ & $\mathrm{NE}$ & $\mathrm{NE}$ \\
\hline \multicolumn{7}{|l|}{ CLuPEIFormes } \\
\hline \multicolumn{7}{|l|}{ Clupeidae } \\
\hline Harengula clupeola (Cuvier, 1829) & Sardinha-cascuda & False Herring, False Pilchard & $\mathrm{Ca}+\mathrm{Br}$ & $\mathrm{NE}$ & $\mathrm{NE}$ & $\mathrm{DD}$ \\
\hline Opisthonema oglinum (Lesueur, 1818) & Sardinha-bandeira & Atlantic Thread Herring & $\mathrm{Ca}+\mathrm{Br}$ & $\mathrm{NE}$ & $\mathrm{NE}$ & NT \\
\hline Sardinella brasiliensis (Steindachner, 1879) & Sardinha, Sardinha-verdadeira & Brazilian Sardinella & $\mathrm{Ca}+\mathrm{Br}+\mathrm{SSWA}$ & $\mathrm{NE}$ & $\mathrm{NE}$ & NFMP \\
\hline \multicolumn{7}{|l|}{ Engraulidae } \\
\hline Anchoa lyolepis (Evermann \& Marsh, 1900) & Manjuba & Shortfinger Anchovy & WA & $\mathrm{NE}$ & $\mathrm{NE}$ & DD \\
\hline Anchoa tricolor (Spix \& Agassiz, 1829) & Manjuba, Irico & Piquitinga Anchovy & SWA & $\mathrm{NE}$ & $\mathrm{NE}$ & DD \\
\hline Anchoviella lepidentostole (Fowler, 1911) & Manjuba & Broadband Anchovy & $\mathrm{Br}$ & $\mathrm{NE}$ & $\mathrm{NE}$ & NT \\
\hline Lycengraulis grossidens (Spix \& Agassiz, 1829) & Manjuba & Atlantic Sabretooth Anchovy & $\mathrm{Ca}+\mathrm{Br}+\mathrm{SSWA}$ & $\mathrm{NE}$ & $\mathrm{NE}$ & $\mathrm{NE}$ \\
\hline \multicolumn{7}{|l|}{ Pristigasteridae } \\
\hline Pellona harroweri (Fowler, 1917) & Sardinha-manteiga & American Coastal Pellona & $\mathrm{Ca}+\mathrm{Br}$ & $\mathrm{NE}$ & $\mathrm{NE}$ & $\mathrm{NE}$ \\
\hline \multicolumn{7}{|l|}{ SILURIFORMES } \\
\hline \multicolumn{7}{|l|}{ Ariidae } \\
\hline Genidens genidens (Cuvier, 1829) & Bagre & Catfish & $\mathrm{Br}$ & LC & $\mathrm{NE}$ & $\mathrm{DD}$ \\
\hline \multicolumn{7}{|l|}{ AuLOPIFORMES } \\
\hline \multicolumn{7}{|l|}{ Synodontidae } \\
\hline Synodus foetens (Linnaeus, 1758) & Peixe-lagarto & Inshore Lizardfish & WA & $\mathrm{NE}$ & $\mathrm{NE}$ & $\mathrm{NE}$ \\
\hline Trachinocephalus myops (Forster, 1801) & Peixe-lagarto & Snakefish & $\mathrm{CT}$ & LC & $\mathrm{NE}$ & $\mathrm{NE}$ \\
\hline \multicolumn{7}{|l|}{ MUGILIFORMES } \\
\hline \multicolumn{7}{|l|}{ Mugilidae } \\
\hline Mugil curema Valenciennes, 1836 & Parati & White Mullet & $\mathrm{TA}+\mathrm{EP}$ & $\mathrm{NE}$ & $\mathrm{NE}$ & DD \\
\hline Mugil liza Valenciennes, 1836 & Tainha & Lebranche Mullet & $\mathrm{Ca}+\mathrm{Br}+\mathrm{SSWA}$ & $\mathrm{NE}$ & $\mathrm{NE}$ & NFMP \\
\hline \multicolumn{7}{|l|}{ GOBIESOCIFORMES } \\
\hline \multicolumn{7}{|l|}{ Gobiesocidae } \\
\hline Gobiesox barbatulus Starks, 1913 & Pregador & Lappetlip Clingfish & $\mathrm{Ca}+\mathrm{Br}$ & $\mathrm{NE}$ & $\mathrm{NE}$ & $\mathrm{NE}$ \\
\hline Gobiesox strumosus Cope, 1870 & Maria-da-toca, Piramangaba & Skilletfish & $W A+E P$ & $\mathrm{NE}$ & $\mathrm{NE}$ & $\mathrm{NE}$ \\
\hline AtHERINIFORMES & & & & & & \\
\hline Atherinopsidae & & & & & & \\
\hline Atherinella brasiliensis (Quoy \& Gaimard, 1825) & Peixe-rei & Brazilian Silversides & $\mathrm{Br}$ & $\mathrm{NE}$ & $\mathrm{NE}$ & $\mathrm{NE}$ \\
\hline CYPRINODONTIFORMES & & & & & & \\
\hline Poeciliidae & & & & & & \\
\hline Poecilia vivipara Bloch \& Schneider, 1801 & Guarú & - & $\mathrm{Ca}+\mathrm{Br}+\mathrm{SSWA}$ & $\mathrm{NE}$ & $\mathrm{NE}$ & $\mathrm{NE}$ \\
\hline BELONIFORMES & & & & & & \\
\hline Belonidae & & & & & & \\
\hline Strongylura marina (Walbaum, 1792) & Agulha & Atlantic Needlefish & WA & LC & $\mathrm{NE}$ & $\mathrm{DD}$ \\
\hline Strongylura timucu (Walbaum, 1792) & Agulha & Timucu & $\mathrm{Ca}+\mathrm{Br}$ & $\mathrm{NE}$ & $\mathrm{NE}$ & $\mathrm{DD}$ \\
\hline Tylosurus acus (Lacepède, 1803) & Agulhão & Agujon Needlefish & $\mathrm{TA}$ & $\mathrm{NE}$ & $\mathrm{NE}$ & $\mathrm{DD}$ \\
\hline Hemiramphidae & & & & & & \\
\hline Hemiramphus brasiliensis (Linnaeus, 1758) & Agulha-preta, Agulhinha & Ballyhoo Halfbeak & TA & $\mathrm{NE}$ & $\mathrm{NE}$ & $\mathrm{DD}$ \\
\hline Hyporhamphus roberti (Valenciennes, 1847) & Agulha & Slender Halfbeak & $\mathrm{Ca}+\mathrm{Br}$ & LC & $\mathrm{NE}$ & $\mathrm{DD}$ \\
\hline Hyporhamphus unifasciatus (Ranzani, 1841) & Peixe-agulha & Common Halfbeak & $\mathrm{Ca}+\mathrm{Br}+\mathrm{SSWA}$ & $\mathrm{NE}$ & $\mathrm{NE}$ & $\mathrm{DD}$ \\
\hline SYNGNATHIFORMES & & & & & & \\
\hline Syngnathidae & & & & & & \\
\hline Cosmocampus elucens (Poey, 1868) & Peixe-cachimbo & Shortfin Pipefish & WA & LC & $\mathrm{NE}$ & $\mathrm{NE}$ \\
\hline Hippocampus reidi Ginsburg, 1933 & Cavalo-marinho & Longsnout Seahorse & WA & $\mathrm{DD}$ & VU & NFMP \\
\hline SCORPAENIFORMES & & & & & & \\
\hline Dactylopteridae & & & & & & \\
\hline Dactylopterus volitans (Linnaeus, 1758) & Coió, Voador & Flying Gurnard & $\mathrm{TA}$ & $\mathrm{NE}$ & $\mathrm{NE}$ & $\mathrm{NE}$ \\
\hline Scorpaenidae & & & & & & \\
\hline
\end{tabular}


Table 1. Continued.

\begin{tabular}{|c|c|c|c|c|c|c|}
\hline Species & Local common names & English common names & Distribution & IUCN & BR & SP \\
\hline \multicolumn{7}{|l|}{ Triglidae } \\
\hline Prionotus punctatus (Bloch, 1793) & Peixe-cabra, Cabrinha & Bluewing Searobin & $\mathrm{Ca}+\mathrm{Br}+\mathrm{SSWA}$ & $\mathrm{NE}$ & $\mathrm{NE}$ & NE \\
\hline \multicolumn{7}{|l|}{ Perciformes } \\
\hline \multicolumn{7}{|l|}{ Centropomidae } \\
\hline Centropomus parallelus Poey, 1860 & Robalo-peva & Fat Snook & $\mathrm{Ca}+\mathrm{Br}$ & $\mathrm{NE}$ & $\mathrm{NE}$ & NT \\
\hline Centropomus undecimalis (Bloch, 1792) & Robalo-flecha & Common Snook & WA & $\mathrm{NE}$ & $\mathrm{NE}$ & NT \\
\hline \multicolumn{7}{|l|}{ Serranidae } \\
\hline Diplectrum formosum (Linnaeus, 1766) & Michole-da-areia & Sand Perch & WA & $\mathrm{NE}$ & $\mathrm{NE}$ & NE \\
\hline Diplectrum radiale (Quoy \& Gaimard, 1824) & Michole-da-areia, Jacundá & Pond Perch & $\mathrm{Ca}+\mathrm{Br}+\mathrm{SSWA}$ & $\mathrm{NE}$ & $\mathrm{NE}$ & NE \\
\hline Epinephelus marginatus (Lowe, 1834) & Garoupa, Garoupa-verdadeira & Dusky Grouper & TA & EN & $C R$ & NFMP \\
\hline Mycteroperca acutirostris (Valenciennes, 1828) & Badejo-mira & Comb Grouper & $\mathrm{Ca}+\mathrm{Br}$ & LC & $\mathrm{NE}$ & NE \\
\hline \multicolumn{7}{|l|}{ Pomatomidae } \\
\hline Pomatomus saltatrix (Linnaeus, 1766) & Anchova & Bluefish & CT & $\mathrm{NE}$ & $\mathrm{NE}$ & NT \\
\hline \multicolumn{7}{|l|}{ Carangidae } \\
\hline Caranx hippos (Linnaeus, 1766)* & Xaréu, Aracimbora & Common Jack, Crevalle Jack & TA & $\mathrm{NE}$ & $\mathrm{NE}$ & NE \\
\hline Caranx latus Agassiz, 1831 & Xerelete & Horse-eye Jack & TA & $\mathrm{NE}$ & $\mathrm{NE}$ & NE \\
\hline Chloroscombrus chrysurus (Linnaeus, 1766) & Palombeta, Carapau & Atlantic Bumper & TA & $\mathrm{NE}$ & $\mathrm{NE}$ & $\mathrm{NE}$ \\
\hline Hemicaranx amblyrhynchus (Cuvier, 1833) & Cabeça dura, Vento-leste & Bluntnose Jack & WA & $\mathrm{NE}$ & $\mathrm{NE}$ & NE \\
\hline Oligoplites saliens (Bloch, 1793) & Guaivira & Castin Leatherjacket & $\mathrm{Ca}+\mathrm{Br}+\mathrm{SSWA}$ & $\mathrm{NE}$ & $\mathrm{NE}$ & NE \\
\hline Oligoplites saurus (Bloch \& Schneider, 1801) & Tibiro de couro & Leatherjacket & $W A+E P$ & $\mathrm{NE}$ & $\mathrm{NE}$ & NE \\
\hline Selene setapinnis (Mitchill, 1815) & Galo & Atlantic Moonfish & WA & $\mathrm{NE}$ & $\mathrm{NE}$ & NT \\
\hline Selene vomer (Linnaeus, 1758) & Galo de penacho & Lookdown & WA & NE & $\mathrm{NE}$ & NE \\
\hline Trachinotus carolinus (Linnaeus, 1766) & Pampo & Florida Pompano & WA & $\mathrm{NE}$ & $\mathrm{NE}$ & NE \\
\hline Trachinotus falcatus (Linnaeus, 1758) & Sernambiguara & Permit & WA & $\mathrm{NE}$ & $\mathrm{NE}$ & NE \\
\hline Trachinotus goodei Jordan \& Evermann, 1896 & Pampo-galhudo & Great Pompano & WA & LC & $\mathrm{NE}$ & NE \\
\hline \multicolumn{7}{|l|}{ Lutjanidae } \\
\hline Lutjanus analis (Cuvier, 1828) & Caranho-vermelho & Mutton Snapper & WA & Vu & $\mathrm{NE}$ & NFMP \\
\hline Lutjanus cyanopterus (Cuvier, 1828) & Caranha & Cubera Snapper & WA & VU & Vu & NFMP \\
\hline Lutjanus synagris (Linnaeus, 1758) & Ariocó, Vermelho-ariocó & Lane Snapper & WA & $\mathrm{NE}$ & $\mathrm{NE}$ & NE \\
\hline \multicolumn{7}{|l|}{ Gerreidae } \\
\hline Diapterus rhombeus (Cuvier, 1829) & Carapeba & Caitipa Mojarra & $\mathrm{Ca}+\mathrm{Br}$ & $\mathrm{NE}$ & $\mathrm{NE}$ & NE \\
\hline Eucinostomus argenteus Baird \& Girard, 1855 & Carapicu & Silver Mojarra & $W A+E P$ & NE & $\mathrm{NE}$ & NE \\
\hline Eucinostomus gula (Quoy \& Gaimard, 1824) & Carapicu & Jenny Mojarra & WA & NE & $\mathrm{NE}$ & $\mathrm{DD}$ \\
\hline Eucinostomus melanopterus (Bleeker, 1863) & Carapicu & Flagfin Mojarra & TA & $\mathrm{NE}$ & $\mathrm{NE}$ & NE \\
\hline Eugerres brasilianus (Cuvier, 1830) & Caratinga & Brazilian Mojarra & WA & $\mathrm{NE}$ & $\mathrm{NE}$ & DD \\
\hline \multicolumn{7}{|l|}{ Haemulidae } \\
\hline Anisotremus surinamensis (Bloch, 1791) & Sargo-beiçudo, Sargo-de-beiço & Black Margate & $\mathrm{Ca}+\mathrm{Br}$ & $\mathrm{NE}$ & $\mathrm{NE}$ & NE \\
\hline Anisotremus virginicus (Linnaeus, 1758) & Salema & Porkfish & $\mathrm{Ca}+\mathrm{Br}$ & $\mathrm{NE}$ & $\mathrm{NE}$ & NE \\
\hline Conodon nobilis (Linnaeus, 1758)* & Roncador & Barred Grunt & $\mathrm{Ca}+\mathrm{Br}$ & $\mathrm{NE}$ & $\mathrm{NE}$ & DD \\
\hline Haemulon steindachneri (Jordan \& Gilbert, 1882) & Corcoroca-boca-larga, Macasso & Chere-chere Grunt & WA+EP & LC & $\mathrm{NE}$ & NE \\
\hline Haemulopsis corvinaeformis (Steindachner, 1868) & Corcoroca legítima, coró-branco & Roughneck Grunt & $\mathrm{Ca}+\mathrm{Br}$ & NE & NE & NE \\
\hline Orthopristis ruber (Cuvier, 1830) & Corcoroca jurumirim, Canguito & Corocoro Grunt & $\mathrm{Ca}+\mathrm{Br}$ & $\mathrm{NE}$ & $\mathrm{NE}$ & NT \\
\hline \multicolumn{7}{|l|}{ Sparidae } \\
\hline Archosargus rhomboidalis (Linnaeus, 1758) & Canhanha, Salema & Sea Bream & WA & LC & $\mathrm{NE}$ & NE \\
\hline Calamus penna (Valenciennes, 1830) & Peixe-pena & Sheepshead Porgy & $\mathrm{Ca}+\mathrm{Br}$ & LC & $\mathrm{NE}$ & $\mathrm{NE}$ \\
\hline Diplodus argenteus (Valenciennes, 1830) & Pargo-branco & Silver Porgy & $\mathrm{Ca}+\mathrm{Br}+\mathrm{SSWA}$ & LC & $\mathrm{NE}$ & $\mathrm{NE}$ \\
\hline \multicolumn{7}{|l|}{ Polynemidae } \\
\hline Polydactylus virginicus (Linnaeus, 1758) & Barbudo, Parati-barbudo & Barbu & WA & $\mathrm{NE}$ & NE & NE \\
\hline \multicolumn{7}{|l|}{ Sciaenidae } \\
\hline Ctenosciaena gracilicirrhus (Metzelaar, 1919) & Pescada cascuda, Goretê & Barbel Drum & $\mathrm{Ca}+\mathrm{Br}$ & $\mathrm{NE}$ & NE & NE \\
\hline Cynoscion jamaicensis (Vaillant \& Bocourt, 1883) & Goete & Jamaica Weakfish & $\mathrm{Ca}+\mathrm{Br}+\mathrm{SSWA}$ & NE & $\mathrm{NE}$ & NT \\
\hline Cynoscion leiarchus (Cuvier, 1830) & Pescada-branca & Smooth Weakfish & $\mathrm{Ca}+\mathrm{Br}$ & $\mathrm{NE}$ & $\mathrm{NE}$ & DD \\
\hline Isopisthus parvipinnis (Cuvier, 1830)* & $\begin{array}{l}\text { Corvina manteiga, Pescada- } \\
\text { mole }\end{array}$ & Bigtooth Corvina & $\mathrm{Ca}+\mathrm{Br}$ & NE & $\mathrm{NE}$ & NE \\
\hline Larimus breviceps Cuvier, 1830 & Oveva & Shorthead Drum & $\mathrm{Ca}+\mathrm{Br}$ & $\mathrm{NE}$ & $\mathrm{NE}$ & $\mathrm{NE}$ \\
\hline Menticirrhus americanus (Linnaeus, 1758) & Papa-terra, Judeu & Southern Kingcroaker & WA & $\mathrm{NE}$ & $\mathrm{NE}$ & NT \\
\hline Micropogonias furnieri (Desmarest, 1823) & Corvina & Whitemouth Croaker & $\mathrm{Ca}+\mathrm{Br}+\mathrm{SSWA}$ & $\mathrm{NE}$ & $\mathrm{NE}$ & NFMP \\
\hline Odontoscion dentex (Cuvier, 1830) & Pescada & Reef Croaker & $\mathrm{Ca}+\mathrm{Br}$ & NE & $\mathrm{NE}$ & DD \\
\hline Umbrina coroides Cuvier, 1830 & Castanha-riscada, Corvina-riscada & Sand Drum & $\mathrm{Ca}+\mathrm{Br}$ & $\mathrm{NE}$ & $\mathrm{NE}$ & $\mathrm{NE}$ \\
\hline \multicolumn{7}{|l|}{ Mullidae } \\
\hline Upeneus parvus Poey, 1852 & Trilha & Dwarf Goatfish & WA & $\mathrm{NE}$ & $\mathrm{NE}$ & $\mathrm{DD}$ \\
\hline
\end{tabular}


Table 1. Continued.

\begin{tabular}{|c|c|c|c|c|c|c|}
\hline Species & Local common names & English common names & Distribution & IUCN & BR & SP \\
\hline \multicolumn{7}{|l|}{ Chaetodontidae } \\
\hline Chaetodon striatus Linnaeus, 1758 & Peixe-borboleta & Banded Butterflyfish & WA & LC & NE & NE \\
\hline \multicolumn{7}{|l|}{ Kyphosidae } \\
\hline Kyphosus incisor (Cuvier, 1831) & Pirajica & Yellow Sea Chub & TA & NE & NE & NE \\
\hline Kyphosus sectatrix (Linnaeus, 1758) & Pirajica & Bermuda Sea Chub & TA & NE & NE & NE \\
\hline \multicolumn{7}{|l|}{ Pomacentridae } \\
\hline Abudefduf saxatilis (Linnaeus, 1758) & Sargentinho, Sinhá-rosa & Sergeant-Major & TA & $\mathrm{NE}$ & NE & NE \\
\hline \multicolumn{7}{|l|}{ Scaridae } \\
\hline Nicholsina usta usta (Valenciennes, 1840) & Budião & Emerald Parrotfish & WA & LC & NE & NE \\
\hline \multicolumn{7}{|l|}{ Uranoscopidae } \\
\hline Astroscopus y-graecum (Cuvier, 1829) & Miracéu & Southern Stargazer & WA & NE & NE & $\mathrm{DD}$ \\
\hline \multicolumn{7}{|l|}{ Labrisomidae } \\
\hline Labrisomus nuchipinnis (Quoy \& Gaimard, 1824) & Guavina, Macaco & Hairy Blenny & TA & LC & NE & NE \\
\hline Malacoctenus delalandii (Valenciennes, 1836) & Macaquino & Brazilian Blenny & $\mathrm{Ca}+\mathrm{Br}$ & LC & NE & NE \\
\hline \multicolumn{7}{|l|}{ Blenniidae } \\
\hline Hypleurochilus fissicornis (Quoy \& Gaimard, 1824) & Maria-da-toca & - & SWA & LC & NE & NE \\
\hline Omobranchus punctatus (Valenciennes, 1836) & - & Muzzled Blenny & - & NE & NE & NE \\
\hline Parablennius pilicornis (Cuvier, 1829) & Maria-da-toca & Ringneck Blenny & TA & LC & NE & NE \\
\hline Scartella cristata (Linnaeus, 1758) & Peixe macaco & Molly Miller & TA & LC & NE & NE \\
\hline \multicolumn{7}{|l|}{ Gobiidae } \\
\hline Bathygobius soporator (Valenciennes, 1837) & Amboré & Frillfin Goby & $\mathrm{TA}+\mathrm{EP}$ & NE & NE & NE \\
\hline Ctenogobius boleosoma (Jordan \& Gilbert, 1882) & Amboré, Rondon & Darter Goby & WA & $\mathrm{NE}$ & NE & NE \\
\hline Ctenogobius smaragdus (Valenciennes, 1837) & Amboré, Maria-da-toca & Emerald Goby & WA & $\mathrm{NE}$ & NE & NE \\
\hline Gobionellus stomatus Starks, 1913* & Amoré & - & $\mathrm{Br}$ & $\mathrm{NE}$ & NE & NE \\
\hline Gobionellus oceanicus (Pallas, 1770) & Amoré & Highfin Goby & WA & $\mathrm{NE}$ & NE & $\mathrm{NE}$ \\
\hline Microgobius meeki Evermann \& Marsh, 1899* & Amborê & - & $\mathrm{Ca}+\mathrm{Br}$ & $\mathrm{NE}$ & NE & $\mathrm{NE}$ \\
\hline \multicolumn{7}{|l|}{ Eleotridae } \\
\hline Butis koilomatodon(Bleeker, 1849) & Barrigudo, Dorminhoco & Mud Sleeper & - & NE & NE & $\mathrm{NE}$ \\
\hline \multicolumn{7}{|l|}{ Ephippidae } \\
\hline Chaetodipterus faber (Broussonet, 1782) & Paru, Enxada & Atlantic Spadefish & WA & NE & NE & $\mathrm{NE}$ \\
\hline \multicolumn{7}{|l|}{ Sphyraenidae } \\
\hline Sphyraena tome Fowler, 1903* & Bicuda, Barracuda & - & SSWA & NE & NE & DD \\
\hline \multicolumn{7}{|l|}{ Trichiuridae } \\
\hline Trichiurus lepturus Linnaeus, 1758 & Peixe-espada & Largehead Hairtail & CT & NE & NE & $\mathrm{NE}$ \\
\hline \multicolumn{7}{|l|}{ Scombridae } \\
\hline $\begin{array}{l}\text { Scomberomorus brasiliensis Collette, Russo \& Zavala- } \\
\text { Camin, } 1978\end{array}$ & Sororoca & Serra Spanish Mackerel & $\mathrm{Ca}+\mathrm{Br}$ & LC & NE & DD \\
\hline \multicolumn{7}{|l|}{ Pleuronectiformes } \\
\hline \multicolumn{7}{|l|}{ Paralichthyidae } \\
\hline Citharichthys arenaceus Evermann \& Marsh, 1900 & Linguado & Sand Whiff & $\mathrm{Ca}+\mathrm{Br}$ & NE & NE & $\mathrm{DD}$ \\
\hline Citharichthys macrops Dresel, 1885 & Linguado & Spotted Whiff & WA & NE & NE & DD \\
\hline Citharichthys spilopterus Günther, 1862 & Linguado, Solha-linguada & Bay Whiff & WA & NE & NE & $\mathrm{DD}$ \\
\hline Etropus crossotus Jordan \& Gilbert, 1882 & Linguado, Solha & Fringed Flounder & WA+EP & NE & NE & $\mathrm{DD}$ \\
\hline Etropus longimanus Norman, 1933 & Linguado & - & SSWA & $\mathrm{NE}$ & NE & DD \\
\hline Syacium papillosum (Linnaeus, 1758) & Linguado & Dusky Flounder & WA & NE & NE & DD \\
\hline Bothidae & & & & & & \\
\hline Bothus ocellatus (Agassiz, 1831) & Linguado & Eyed Flounder & WA & NE & NE & $\mathrm{DD}$ \\
\hline Achiridae & & & & & & \\
\hline Achirus lineatus (Linnaeus, 1758) & Linguado, Solha & Lined Sole & $\mathrm{Ca}+\mathrm{Br}+\mathrm{SSWA}$ & NE & NE & DD \\
\hline Cynoglossidae & & & & & & \\
\hline Symphurus tessellatus (Quoy \& Gaimard, 1824) & Língua-de-mulata & Tessellated Tonguefish & $\mathrm{Ca}+\mathrm{Br}+\mathrm{SSWA}$ & NE & NE & DD \\
\hline TETRAODONTIFORMES & & & & & & \\
\hline Monacanthidae & & & & & & \\
\hline Stephanolepis hispidus (Linnaeus, 1766) & Peixe-porco & Planehead Filefish & TA & NE & NE & $\mathrm{NE}$ \\
\hline Tetraodontidae & & & & & & \\
\hline Lagocephalus laevigatus (Linnaeus, 1766) & Baiacu & Smooth Puffer & WA & LC & NE & DD \\
\hline Sphoeroides greeleyi Gilbert, 1900 & Baiacu & Green Puffer & $\mathrm{Ca}+\mathrm{Br}$ & LC & NE & $\mathrm{DD}$ \\
\hline Sphoeroides spengleri (Bloch, 1785) & Baiacu & Bandtail Puffer & WA & LC & NE & $\mathrm{DD}$ \\
\hline Sphoeroides testudineus (Linnaeus, 1758) & Baiacu & Checkered Puffer & WA & LC & NE & DD \\
\hline Diodontidae & & & & & & \\
\hline Chilomycterus spinosus (Linnaeus, 1758) & Baiacu, Baiacu-de-espinho & Brown Burrfish & $\mathrm{Br}+\mathrm{SSWA}$ & NE & NE & $\mathrm{DD}$ \\
\hline
\end{tabular}




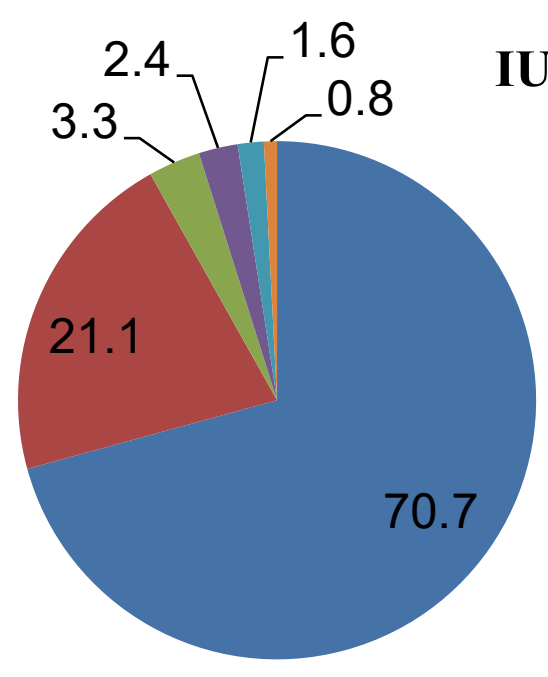

IUCN
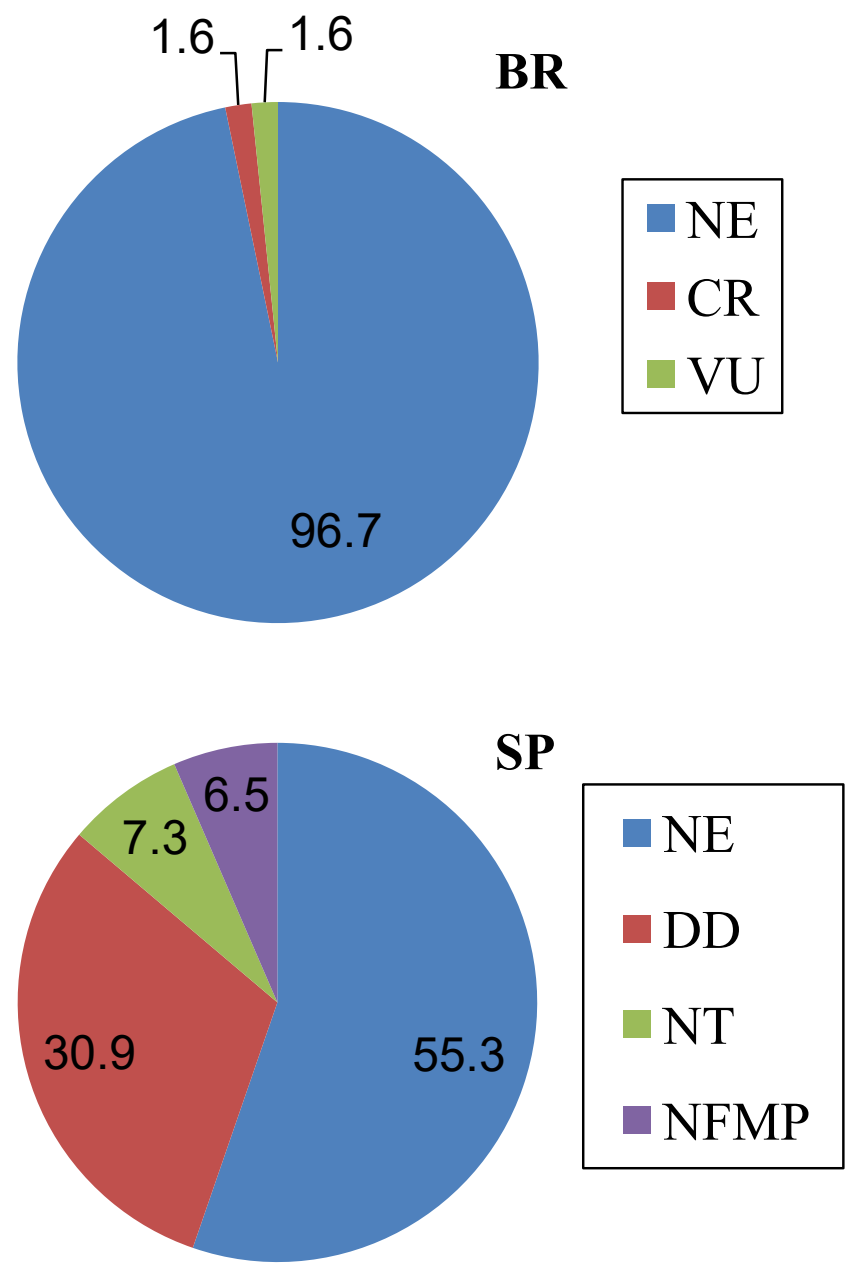

Figure 3. Relative frequency of the conservation status of species recorded in Araçá Bay (total richness = 126) in the IUCN Red List (IUCN 2014), in the federal list (BR; Brasil 2014), and in the state list (SP; Governo do Estado de São Paulo 2014). IUCN: NE - Not Evaluated; LC -Least Concern; DD - Data Deficient; VU - Vulnerable; NT - Near Threatened; EN - Endangered. BR: NE - Not Evaluated; VU - Vulnerable; CR -Critically Endangered. SP: NE - Not Evaluated; DD - Data Deficient; NT - Near Threatened; NFMP - Needed Fisheries Management Plan. For the IUCN classification criteria see'The IUCN Red List of Threatened Species' (IUCN 2014), for the federal classification criteria see the Ordinance of the Ministry of Environment $n^{\circ} 445$, of 17 December 2014 (Brasil 2014) and for the state classification criteria see the Decree No 60.133 of 7 February 2014 (Governo do Estado de São Paulo 2014).
Janeiro (Araújo et al. 2002), and Flamengo Cove, Ubatuba (Mattox et al. 2014). In Araçá Bay, as in those areas, the richest families in terms of species were Sciaenidae, Haemulidae, Paralichthydae, and Carangidae. In fact, such families, especially Carangidae and Sciaenidae, are commonly found throughout the Brazilian coast (Vazzoler et al. 1999).

Although potential differences in fish species composition may be due to differences in the sampling processes and protocols, a comparison of the Araçá Bay species composition to other southeastern Brazilian coastal ecosystems might be informative. The richness of Araçá Bay (126 spp.) is greater than on the Ubatuba shelf, where a total of 79 and 111 species were reported by Rocha and Rossi-Wongtschowski (1998), and Costa (2014), respectively. A lesser richness than in Araçá Bay was also found in adjacent regions of the SSC by RossiWongtschowski et al. (2008), and Gibran and Moura (2012), who found 65 and 68 species, respectively. The high species richness encountered in the present study may be due to the varied (nine) gear types used, while the other studies used only one. These comparisons emphasize the importance of combining different sampling methods to attain a more complete picture of the species composition in fish assemblages (Olin and Malinen 2003). It is worth pointing out that Araçá Bay shares approximately half of its fish fauna with the SSC (43\%, Lamas 2015), which suggests a considerable connectivity of species.

A bottom trawl survey carried out 20 years ago in the outer sublittoral of the Araçá Bay recorded the following species not recorded in the present study: Chirocentrodon bleekerianus (Poey, 1867), Cyclopsetta chittendeni Bean, 1895, Dules auriga Cuvier, 1829, Epinephelus morio (Valenciennes, 1828), Hyporthodus nigritus (Holbrook, 1855), Ogcocephalus verpertilio (Linnaeus, 1758), Porichthys porosissimus (Cuvier, 1829), Sphoeroides tyleri Shipp, 1972 and Trinectes paulistanus (Miranda Ribeiro, 1915) (Muto et al. 2000). The absence of these species in our samples may be due to sampling effort but also potential differences in fish community between 1994 and 2016. For this reason, these species have not been included in the present checklist.

Most of the fish fauna of Araçá Bay is either widely distributed throughout the subtropical and tropical Western Atlantic, or more restricted to the tropical region (i.e., occurring in both Caribbean and Brazilian Province), and there are also species more related to the temperate region (i.e., southern South West Atlantic). The geographic distribution pattern of Araçá fishes reflects the bay's location in the Argentinian zoogeographic province, which extends from Cabo Frio, Rio de Janeiro, to the Valdés Peninsula, Argentina (Caires 2014). The Argentinian zoogeographic province is a large faunistic transition zone, where tropical and 
temperate fauna meets, but also has many endemic species (Caires 2014). Three of the endemic fish species, Dasyatis hypostigma Santos \& Carvalho, 2004, Sardinella brasiliensis, and Sphyraena tome Fowler, 1903, were recorded in Araçá Bay.

With the expansion of the São Sebastião Port, habitat will be lost within the bay and a substantial increase in pollution from oil spills and sewage may be expected. These are threats to fish populations within the bay. We found legally endangered species, such as Epinephelus marginatus and Gymnura altavela, in Araçá Bay, which means that measures should be taken to protect their populations during and after the port expansion, as determined by federal legislation (Brasil 2014). Our results also found species in need of fishery management, such as Hippocampus reidi, Lutjanus analis, Lutjanus cyanopterus, Micropogonias furnieri, Mugil liza, Rhinobatos percellens and Sardinella brasiliensis, which emphasizes the inclusion of the bay in the regional fisheries planning.

Also important is the presence of two non-native species, O. punctatus and B. koilomatodon. These species were likely introduced into the bay with ballast water and as part of the biofouling communities on cargo ships that dock in the São Sebastião Port. With the port expansion, a substantial increase in ship traffic is expected, which increases the probability of new introductions. Non-native species pose a threat to native species by out-competing for habitat and resources (Contente et al. 2015; Contente et al. 2016).

Our study also determined that the conservation status of many species found in Araçá Bay, and elsewhere along the Brazilian coast, remains unevaluated. This may be a consequence of the paucity of biological and ecological data on these species, which are needed for ranking status. We found that species often received a different ranking in the state and federal lists, and that the state list classifies many more species as threatened than the federal one. This highlights the disparity of methods and criteria used (Gärdenfors 2001; Possingham et al. 2002).

This study provides the first checklist of the fish species of the Araça Bay, with remarks on their distribution and conservation, which can be an important tool for future environmental impact, biogeographical and macroecological studies.

\section{ACKNOWLEDGEMENTS}

We thank Alexandre Arackawa for editing the photos, the Laboratory of Ichthyology and Growth's team, fishermen Neemias Borges and Marcelo Alves for fieldwork cooperation, the São Paulo Research Foundation (FAPESP) for financial support (RAL grant number: 13/06938-0; CLDBRW grant number: 2011/50317-5; RFC grant number: 2013/19435-7), the Centre for
Marine Biology of the University of São Paulo for the use of laboratories, and the Chico Mendes Institute for Biodiversity Conservation for allowing the capture, storage and transport of the fish (permit numbers 5189, 5218, 5553, 5866, and 6104)

\section{LITERATURE CITED}

Amaral, A.C.Z. and S.A.H. Nallin. 2011. Biodiversidade e ecossistemas bentônicos marinhos do Litoral Norte de São Paulo, Sudeste do Brasil. Campinas: UNICAMP/IB.

Amaral, A.C.Z., A.E. Migotto, A. Turra and Y. Schaeffer-Novelli. 2010. Araçá: biodiversidade, impactos e ameaças. Biota Neotropica 10(1): 219-264. doi: 10.1590/S1676-06032010000100022

Araújo, F.G., M.C.C. de Azevedo, M. de A. Silva, A.L.M. Pessanha, I.D. Gomes and A.G. da Cruz-Filho. 2002. Environmental influences on the demersal fish assemblages in the Sepetiba Bay, Brazil. Estuaries 25(3): 441-450. doi: 10.1007/BF02695986

Arruda, E.P. and A.C.Z.Amaral. 2003. Spatial distribution of Mollusks in the intertidal zone of sheltered beaches in southeastern of Brazil. Revista Brasileira de Zoologia 20(2): 291-300. doi: 10.1590/S0101-81752003000200020

Brasil. 2014. Ministério do Meio Ambiente - MMA. Portaria n ${ }^{\circ} 445$, de 17 de dezembro de 2014. Reconhecer como espécies de peixes e invertebrados aquáticos da fauna brasileira ameaçadas de extinção aquelas constantes da "Lista Nacional Oficial de Espécies da Fauna Ameaçadas de Extinção-Peixes e Invertebrados Aquáticos".

Brenha-Nunes, M.R., R.F. Contente and C.L.D.B. Rossi-Wongtschowski. 2016. A protocol for measuring spatial variables in soft-sediment tide pools. Zoologia 33(2): 1-4. doi: 10.1590/ S1984-4689zool-20150165

Caires, R.A. 2014. Biogeografia dos Peixes Marinhos do atlântico sul ocidental: Padrões e Processos. Arquivos de Zoologia 45(esp.): 5-24. doi: 10.11606/issn.2176-7793.v45iespp5-24

Carpenter, K.E. 2002a. The living marine resources of the Western Central Atlantic. Volume 1: Introduction, mollusks, crustaceans, hagfishes, sharks, batoid fishes, and chimaeras. Rome: FAO. 1-600 pp.

Carpenter, K.E. 2002b. The living marine resources of the Western Central Atlantic. Volume 2: Bony fishes part 1 (Acipenseridae to Grammatidae). Rome: FAO. 601-1374 pp.

Carpenter, K.E. 2002c. The living marine resources of the Western Central Atlantic. Volume 3: Bony fishes part 2 (Opistognathidae to Molidae), sea turtles and marine mammals. Rome: FAO. 1375-2127 pp.

Consultoria Paulista de Estudos Ambientais. 2011. Relatório de Impacto Ambiental - RIMA: Plano Integrado Porto Cidade (PIPC). São Sebastião: Companhia Docas de São Sebastião. 52 pp. http://www.portodesaosebastiao.com.br/pt-br/rima/ EIA_Rima_Porto_de_Sao_Sebastiao.pdf

Contente, R.F., M.R. Brenha-Nunes, C.C. Siliprandi, R.A. Lamas and V.R.M. Conversani. 2015. Occurrence of the non-indigenous Omobranchus punctatus (Bleniidae) on the São Paulo coast, south-eastern Brazil. Marine Biodiversity Records 8(73): 1-4. doi: 10.1017/S175526721500055X

Contente, R.F., M.R. Brenha-Nunes, C.C. Siliprandi, R.A. Lamas and V.R.M. Conversani. 2016. A new record of the non-native fish species Butis koilomatodon (Bleeker 1849) (Teleostei: Eleotridae) for southeastern Brazil. Biotemas 29(2): 113-118. doi: 10.5007/2175-7925.2016v29n2p113

Corte, G.N., L.Q. Yokoyama and A.C.Z. Amaral. 2014. An attempt to extend the Habitat Harshness Hypothesis to tidal flats: a case study of Anomalocardia brasiliana (Bivalvia: Veneridae) reproductive biology. Estuarine, Coastal and Shelf Science 
150(A): 136-141. doi: 10.1016/j.ecss.2013.12.007

Costa, M.R. 2014. Composição e estrutura da comunidade de peixes em duas praias sob o mesmo tipo de pressão antrópica, litoral de São Paulo, Brasil. UNISANTA BioScience 3(3): 122-142. http:// periodicos.unisanta.br/index.php/bio/article/view/219

Eschmeyer, W.N. 2014. Catalog of fishes: genera, species, references. Accessed at http://research.calacademy.org/research/ichthyology/ catalog/fishcatmain.asp, 15 December 2014.

Figueiredo, J.L. 1977. Manuais de peixes marinhos do sudeste do Brasil: volume 1. São Paulo: Museu de Zoologia da Universidade de São Paulo. 104 pp.

Figueiredo, J.L. and N.A. Menezes. 1978. Manuais de peixes marinhos do sudeste do Brasil: volume 2. São Paulo: Museu de Zoologia da Universidade de São Paulo. 110 pp.

Figueiredo, J.L. and N.A. Menezes. 1980. Manuais de peixes marinhos do sudeste do Brasil: volume 3. São Paulo: Museu de Zoologia da Universidade de São Paulo. 90 pp.

Figueiredo, J.L. and N.A. Menezes. 2000. Manuais de peixes marinhos do sudeste do Brasil: volume 6. São Paulo: Museu de Zoologia da Universidade de São Paulo. 116 pp.

Gärdenfors, U. 2001. Classifying threatened species at national and global levels. Trends in Ecology and Evolution 16(9): 511-516. doi: 10.1016/S0169-5347(01)02214-5

Gibran, F.Z. and R.L. Moura. 2012. The structure of rocky reef fish assemblages across a nearshore to coastal islands' gradient in southeastern Brazil. Neotropical Ichthyology 10(2): 369-382. doi: 10.1590/S1679-62252012005000013

Gorman, D., C.E. Sikinger and A. Turra. 2015. Spatial and temporal variation in the predation risk for hermit crabs in a subtropical bay. Journal of Experimental Marine Biology and Ecology 462: 98-104. doi: 10.1016/j.jembe.2014.10.009

Governo do Estado de São Paulo. Decreto No 60.133, de 7 de fevereiro de 2014. Declara as Espécies da Fauna Silvestre Ameaçadas de extinção, as Quase Ameaçadas e as deficientes em dados para avaliação no Estado de São Paulo e dá providências correlatas. 2014.

Gubitoso, S., W. Duleba, A.C. Teodoro, S.M. Prada, M.M. Rocha, C.C. Lamparelli, J.E. Bevilacqua and D.O. Moura. 2008. Estudo geoambiental da região circunjacente ao emissário submarino de esgoto do Araçá, São Sebastião (SP). Revista Brasileira de Geociências 38(3): 467-475. http://ppegeo.igc.usp.br/scielo. php?script=sci_arttext $\&$ pid $=$ S0375-75362008000500004\&lng =pt\&nrm=iso

Hellmann, J.J. and G.W. Fowler. 1999. Bias, precision, and accuracy of four measures of species richness. Ecological Applications 9(3): 824-834. doi: 10.1890/1051-0761

IUCN (International Union for the Conservation of Nature). 2014. IUCN Red List of threatened species. Version 2014.3. Accessed at http://www.iucnredlist.org, 5 December 2014.

Lamas, R.A. 2015. Banco de dados biológicos e ecológicos da ictiofauna da Baía do Araçá, São Sebastião (SP) [unpublished report]. São Paulo: Instituto Oceanográfico, Universidade de São Paulo. 56 pp.

Mace, G.M. 2004. The role of taxonomy in species conservation. Philosophical Transactions of the Royal Society 359: 711-719. doi: 10.1098/rstb.2003.1454

Mattox, G.M.T., G.F. Gondolo and P.T.M. Cunningham. 2014. Longterm variation in the ichthyofauna of Flamengo Cove, Ubatuba São Paulo. Arquivos de Zoologia 45(esp.): 51-61. doi: 10.11606/ issn.2176-7793.v45iespp51-61

Menezes, N.A. and J.L. Figueiredo. 1980. Manuais de peixes marinhos do sudeste do Brasil: volume 4. São Paulo: Museu de Zoologia da Universidade de São Paulo. 96 pp.

Menezes, N.A., J.L. Figueiredo. 1985. Manuais de peixes marinhos do sudeste do Brasil: volume 5. São Paulo: Museu de Zoologia da Universidade de São Paulo.105 pp.

Migotto, A.E., C.G. Tiago and A.R.M. Magalhães. 1993. Malacofauna marinha da região costeira do Canal de São Sebastião, SP, Brasil: Gastropoda, Bivalvia, Polyplacophora e Scaphopoda. Boletim do Instituto oceanográfico 41(1/2): 13-27. doi: 10.1590/S037355241993000100002

Muto, E.Y., L.S.H. Soares and C.L.D.B. Rossi-Wongtschowski. 2000. Demersal fish assemblages off São Sebastião, southeastern Brazil: structure and environmental conditioning factors (summer 1994). Revista Brasileira de Oceanografia 48(1): 9-27. doi: 10.1590/S1413-77392000000100002

Olin, M. and T. Malinen. 2003. Comparison of gillnet and trawl in diurnal fish community sampling. Hydrobiologia 506-509: 443-449. doi: 10.1023/B:HYDR.0000008545.33035.c4

Passos, A.C., R.F. Contente, C.C.V. de Araujo, F.A.L. de M. Daros, H.L. Spach, V. Abilhôa and L.F. Fávaro. 2012. Fishes of Paranaguá Estuarine Complex, South West Atlantic. Biota Neotrópica 12(3): 226-238. doi: 10.1590/S1676-06032012000300022

Petracco, M., R.M. Camargo, D.T. Tardelli and A. Turra. 2013. Population biology of the gastropod Olivella minuta (Gastropoda, Olividae) on two sheltered beaches in southeastern Brazil. Estuarine, Coastal and Shelf Science 150(A): 149-156. doi: 10.1016/j. ecss.2013.10.015

Possingham, H.P., S.J. Andelmanb, M.A. Burgmanc, R.A. Medellínd, L.L. Mastere and D.A. Keithf. 2002. Limits to the use of threatened species lists. Trends in Ecology and Evolution 17(11): 503-507. doi: 10.1016/S0169-5347(02)02614-9

Rocha, G.R.A. and C.L.D.B. Rossi-Wongtschowski. 1998. Demersal fish community on the inner shelf of Ubatuba, southeastern Brazil. Revista Brasileira de Oceanografia 46(2): 93-109. doi: 10.1590/S1413-77391998000200001

Rossi-Wongtschowski, C.L.D.B., L.S.H. Soares and E.Y. Muto. 2008. Ictiofauna; pp. 381-403, in: A.M.S. Pires-Vanin (ed.). Oceanografia de um ecossistema subtropical: plataforma de São Sebastião, SP. São Paulo: Editora da Universidade de São Paulo.

Rossi-Wongtschowski, C.L.D.B., C.C. Siliprandi and R.F Contente. 2015. Peixes da Baía do Araçá, São Sebastião - SP - Brasil. São Paulo: Uhôa Cintra Comunicação Visual e Arquitetura. 16 pp.

Silveira, R.B. 2011. Registros de cavalos-marinhos (Syngnathidae: Hippocampus) ao longo da costa brasileira. Oecologia Australis 15(2): 316-325. doi: 10.4257/oeco.2011.1502.09

Silveira, L.F., B. de M. Beisiegel, F.F. Curcio, P.H. Valdujo, M. Dixo, V.K. Verdade, G.M.T. Mattox and P.T.M. Cunningham. 2010. Para que servem os inventários de fauna? Estudos Avançados 24(68): 173-178. doi: 10.1590/S0103-40142010000100015

Teodoro, A.C., W. Duleba and S. Gubitoso. 2011. Estudo multidisciplinar (geoquímica e associações de foraminíferos) para caracterizar e avaliar intervenções antrópicas na Baía do Araçá, Canal de São Sebastião, SP. Geologia USP. Série Científica 11(1): 113-136. doi: 10.5327/Z1519-874X2011000100007

Vazzoler, A.E.A. de M., L.S.H. Soares and P.T.M. Cunningham. 1999. Ictiofauna da costa brasileira; pp. 424-467, in: R.H. LoweMcconnell (ed.). Estudos ecológicos de comunidades de peixes tropicais. (A.E.A. de M. Vazzoler, A.A. Agostinho and P.T.M. Cunningham, translators). São Paulo: Editora da Universidade de São Paulo.

Zanardi, E., M.C. Bícego, L.B. de Miranda and R.R. Weber. 1999. Distribution and origin of hydrocarbons in water and sediment in São Sebastião, SP, Brazil. Marine Pollution Bulletin 38(4): 261-267. doi: 10.1016/S0025-326X(98)90143-6

Author contributions: The species data search was performed by RAL; all authors collected the data and prepared the manuscript.

Received: 18 August 2015

Accepted: 12 September 2016

Academic editor: Osmar J. Luiz

APPENDIX 
Table A1. Voucher number of specimens deposited in the Zoological Museum of the University of São Paulo. Species are in alphabetic order.

\begin{tabular}{|c|c|}
\hline Voucher number & Species \\
\hline MZUSP 118862 & Abudefduf saxatilis \\
\hline MZUSP 118863 & Achirus lineatus \\
\hline MZUSP 118864 & Albula vulpes \\
\hline MZUSP 118865 & Anchovia clupeoides \\
\hline MZUSP 118866 & Anchoviella lepidentostole \\
\hline MZUSP 118867 & Anchoa lyoleps \\
\hline MZUSP 118868 & Anisotremus surinamensis \\
\hline MZUSP 118869 & Anchoa tricolor \\
\hline MZUSP 118870 & Anisotremus virginicus \\
\hline MZUSP 118871 & Archosargus rhomboidalis \\
\hline MZUSP 118872 & Astroscopus y-graecum \\
\hline MZUSP 118873 & Atherinella brasiliensis \\
\hline MZUSP 118874 & Bathygobius soporator \\
\hline MZUSP 118875 & Bothus ocellatus \\
\hline MZUSP 118876 & Caranx latus \\
\hline MZUSP 118877 & Calamus penna \\
\hline MZUSP 118878 & Centropomus parallelus \\
\hline MZUSP 118879 & Centropomus undecimalis \\
\hline MZUSP 118880 & Chloroscombrus chrysurus \\
\hline MZUSP 118881 & Chaetodipterus faber \\
\hline MZUSP 118882 & Chilomycterus spinosus \\
\hline MZUSP 118883 & Chaetodon striatus \\
\hline MZUSP 118884 & Citharichthys arenaceus \\
\hline MZUSP 118885 & Citharichthys macrops \\
\hline MZUSP 118886 & Citharichthys spilopterus \\
\hline MZUSP 118887 & Cosmocampus elucens \\
\hline MZUSP 118888 & Ctenogobius boleosoma \\
\hline MZUSP 118889 & Ctenosciaena gracilicirrhus \\
\hline MZUSP 118890 & Ctenogobius smaragdus \\
\hline MZUSP 118891 & Cynoscion jamaiscensis \\
\hline MZUSP 118892 & Cynoscion leiarchus \\
\hline MZUSP 118893 & Dasyatis guttata \\
\hline MZUSP 118894 & Dasyatis hypostigma \\
\hline MZUSP 118895 & Dactylopterus volitans \\
\hline MZUSP 118896 & Diplodus argenteus \\
\hline MZUSP 118897 & Diplectrum formosum \\
\hline MZUSP 118898 & Diplectrum radiale \\
\hline MZUSP 118899 & Diapterus rhombeus \\
\hline MZUSP 118900 & Elops saurus \\
\hline
\end{tabular}

\begin{tabular}{|c|c|}
\hline Voucher number & Species \\
\hline MZUSP 118901 & Epinephelus marginatus \\
\hline MZUSP 118902 & Etropus crossotus \\
\hline MZUSP 118903 & Etropus longimanus \\
\hline MZUSP 118904 & Eucinostomus argenteus \\
\hline MZUSP 118905 & Eugerres brasilianus \\
\hline MZUSP 118906 & Eucinostomus gula \\
\hline MZUSP 118907 & Eucinostomus melanopterus \\
\hline MZUSP 118908 & Genidens genidens \\
\hline MZUSP 118909 & Gobiesox barbatulus \\
\hline MZUSP 118910 & Gobionellus oceanicus \\
\hline MZUSP 118911 & Gobiesox strumosus \\
\hline MZUSP 118912 & Gymnothorax occelatus \\
\hline MZUSP 118913 & Harengula clupeola \\
\hline MZUSP 118914 & Haemulopsis corvinaeformis \\
\hline MZUSP 118915 & Haemulon steindachneri \\
\hline MZUSP 118916 & Hemicaranx amblyrhynchus \\
\hline MZUSP 118917 & Hemiramphus brasiliensis \\
\hline MZUSP 118918 & Hipoccampus reidi \\
\hline MZUSP 118919 & Hypleurochilus fissicornis \\
\hline MZUSP 118920 & Hyporhamphus roberti \\
\hline MZUSP 118921 & Hyporhamphus unifasciatus \\
\hline MZUSP 118922 & Kyphosus incisor \\
\hline MZUSP 118923 & Kyphosus sectatrix \\
\hline MZUSP 118924 & Larimus breviceps \\
\hline MZUSP 118925 & Lagocephalus laevigatus \\
\hline MZUSP 118926 & Labrisomus nuchipinnis \\
\hline MZUSP 118927 & Lutjanus analis \\
\hline MZUSP 118928 & Lutjanus cyanopterus \\
\hline MZUSP 118929 & Lutjanus synagris \\
\hline MZUSP 118930 & Lycengraulis grossidens \\
\hline MZUSP 118931 & Malacoctenus delalandii \\
\hline MZUSP 118932 & Menticirrhus americanus \\
\hline MZUSP 118933 & Micropogonias furnieri \\
\hline MZUSP 118934 & Mugil curema \\
\hline MZUSP 118935 & Mugil hospes \\
\hline MZUSP 118936 & Mugil incilis \\
\hline MZUSP 118937 & Mugil liza \\
\hline MZUSP 118938 & Mycteroperca acutirostris \\
\hline MZUSP 118939 & Myrophis punctatus \\
\hline
\end{tabular}

\begin{tabular}{|c|c|}
\hline Voucher number & Species \\
\hline MZUSP 118940 & Nicholsina usta usta \\
\hline MZUSP 118941 & Odontoscion dentex \\
\hline MZUSP 118942 & Oligoplites saliens \\
\hline MZUSP 118943 & Oligoplites saurus \\
\hline MZUSP 118944 & Ophichthus gomesii \\
\hline MZUSP 118945 & Opisthonema ogliunum \\
\hline MZUSP 118946 & Orthopristis ruber \\
\hline MZUSP 118947 & Parablennius pilicornis \\
\hline MZUSP 118948 & Pellona harroweri \\
\hline MZUSP 118949 & Pomatomus saltatrix \\
\hline MZUSP 118950 & Polydactylus virginicus \\
\hline MZUSP 118951 & Poecillia vivipara \\
\hline MZUSP 118952 & Prionotus punctatus \\
\hline MZUSP 118953 & Rhinobatos percellens \\
\hline MZUSP 118954 & Sardinella brasiliensis \\
\hline MZUSP 118955 & Scomberomorus brasiliensis \\
\hline MZUSP 118956 & Scartella cristata \\
\hline MZUSP 118957 & Scorpaena brasiliensis \\
\hline MZUSP 118958 & Scorpaena plumieri \\
\hline MZUSP 118959 & Selene setapinnis \\
\hline MZUSP 118960 & Selene vomer \\
\hline MZUSP 118961 & Sphoeroides greeleyi \\
\hline MZUSP 118962 & Sphoeroides spengleri \\
\hline MZUSP 118963 & Sphoeroides testudineus \\
\hline MZUSP 118964 & Stephanolepis hispidus \\
\hline MZUSP 118965 & Strongylura marina \\
\hline MZUSP 118966 & Strongylura sp \\
\hline MZUSP 118967 & Strongylura timucu \\
\hline MZUSP 118968 & Synodus foetens \\
\hline MZUSP 118969 & Syacium papillosum \\
\hline MZUSP 118970 & Symphurus tesselatus \\
\hline MZUSP 118971 & Trachinotus carolinus \\
\hline MZUSP 118972 & Trachinotus falcatus \\
\hline MZUSP 118973 & Trachinotus goodei \\
\hline MZUSP 118974 & Trichiurus lepturus \\
\hline MZUSP 118975 & Trachinocephalus myops \\
\hline MZUSP 118976 & Tylosurus acus \\
\hline MZUSP 118977 & Umbrina coroides \\
\hline MZUSP 118978 & Upeneus parvus \\
\hline
\end{tabular}

